University of Wollongong

Research Online

Faculty of Engineering and Information

Faculty of Engineering and Information

Sciences - Papers: Part A

Sciences

$1-1-2015$

\title{
Molecular dynamics simulation on generalized stacking fault energies of FCC metals under preloading stress
}

\author{
Liang Zhang \\ University of Wollongong, Iz592@uowmail.edu.au \\ Cheng Lu \\ University of Wollongong, chenglu@uow.edu.au \\ A Kiet Tieu \\ University of Wollongong, ktieu@uow.edu.au \\ Xing Zhao \\ University of Wollongong, xz920@uowmail.edu.au \\ Linqing Pei \\ University of Wollongong, Ip115@uowmail.edu.au
}

See next page for additional authors

Follow this and additional works at: https://ro.uow.edu.au/eispapers

Part of the Engineering Commons, and the Science and Technology Studies Commons

Research Online is the open access institutional repository for the University of Wollongong. For further information contact the UOW Library: research-pubs@uow.edu.au 


\title{
Molecular dynamics simulation on generalized stacking fault energies of FCC metals under preloading stress
}

\author{
Abstract \\ Molecular dynamics (MD) simulations are performed to investigate the effects of stress on generalized \\ stacking fault (GSF) energy of three fcc metals ( $\mathrm{Cu}, \mathrm{Al}$, and $\mathrm{Ni}$ ). The simulation model is deformed by \\ uniaxial tension or compression in each of [111], [11-2], and [1-10] directions, respectively, before shifting \\ the lattice to calculate the GSF curve. Simulation results show that the values of unstable stacking fault \\ energy (үusf), stable stacking fault energy ( $\gamma s f)$, and unstable twin fault energy (үutf) of the three \\ elements can change with the preloaded tensile or compressive stress in different directions. The ratio of \\ Ysf/Yusf, which is related to the energy barrier for full dislocation nucleation, and the ratio of yutf/yusf, \\ which is related to the energy barrier for twinning formation are plotted each as a function of the \\ preloading stress. The results of this study reveal that the stress state can change the energy barrier of \\ defect nucleation in the crystal lattice, and thereby can play an important role in the deformation \\ mechanism of nanocrystalline material.
}

\section{Keywords}

under, preloading, stress, energies, dynamics, fault, simulation, stacking, generalized, molecular, fcc, metals

Disciplines

Engineering | Science and Technology Studies

\section{Publication Details}

Zhang, L., Lu, C., Tieu, K., Zhao, X., Pei, L. \& Michal, G. (2015). Molecular dynamics simulation on generalized stacking fault energies of FCC metals under preloading stress. Chinese Physics B, 24 (8), 088106-1-088106-8.

\section{Authors}

Liang Zhang, Cheng Lu, A Kiet Tieu, Xing Zhao, Linqing Pei, and Guillaume Michal 


\title{
Molecular Dynamics Simulation on Generalized Stacking Fault Energies of FCC Metals under Preloading Stress
}

\author{
Liang Zhang, Cheng Lu, Kiet Tieu, Xing Zhao, Linqing Pei, Guillaume Michal \\ School of Mechanical, Materials and Mechatronic Engineering, University of Wollongong, \\ Wollongong, NSW 2522, Australia
}

\begin{abstract}
Molecular dynamics (MD) simulations were performed to investigate the effect of stress on generalized stacking fault (GSF) energy of three fcc metals $(\mathrm{Cu}, \mathrm{Al}$ and $\mathrm{Ni}$ ). The simulation model was deformed by uniaxial tension or compression in [1 1

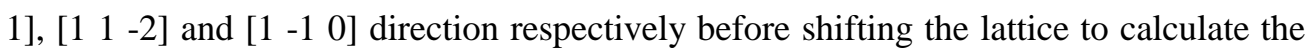
GSF curve. Simulation results show that, the unstable stacking fault energy $\left(\gamma_{\text {usf }}\right)$, stable stacking fault energy $\left(\gamma_{\mathrm{sf}}\right)$ and unstable twin fault energy $\left(\gamma_{\mathrm{utf}}\right)$ of the three elements can change with the preloaded tensile or compressive stress in different directions. The ratio of $\gamma_{\mathrm{sf}} / \gamma_{\mathrm{usf}}$, which is associated with the energy barrier for full dislocation nucleation and the ratio of $\gamma_{\mathrm{utf}} / \gamma_{\mathrm{usf}}$, which is related to the energy barrier for twinning formation were plotted as a function of the preloading stress. The results of this study revealed that the stress state can change the energy barrier of defects nucleation in the crystal lattice, and thereby can play an important role in the deformation mechanisms of nanocrystalline materials.
\end{abstract}

Keywords: Molecular dynamics, EAM, Generalized stacking fault

PACS: 81.07.Nb, 81.07.Bc, 81.40.Vw, 81.40.Jj

\section{Introduction}

The mechanisms of plastic deformation of nanocrystalline materials have been widely studied because of their proved superior functional and mechanical properties. ${ }^{1-4}$ The plastic deformation of conventional coarse-grained materials is mainly accommodated by dislocation nucleation and their motion in the interior of grains. However, nanoscale confinement severely limits the operation of traditional dislocation generation mechanisms in nanocrystalline materials. Both experiment $^{5-7}$ and molecular dynamics (MD) simulations ${ }^{8-10}$ have reported a deviation from traditional Hall-Petch constitutive behavior. Many research works ${ }^{11-14}$ indicated that the dislocation activities in the interior of grains lessen when the average grain size is less than 100 $\mathrm{nm}$, whereas mechanisms mediated by the grain boundary (GB) become dominant. For example, small grain size can result in heterogeneous nucleation and emission of dislocations from GBs ${ }^{15-17}$. Yamakov et al. ${ }^{18}$ proposed a deformation mechanism map that described the transition from dislocation-driven to GB-mediated plastic deformation based on the splitting distance between partial dislocations and the stacking fault energy $\gamma_{\mathrm{sf}}$. Van Swygenhoven and coworkers ${ }^{19}$ revealed that $\gamma_{\mathrm{sf}}$ alone cannot capture the important physics of the nucleation of partial dislocations from GBs, a correct interpretation of the nature of slip in nanocrystalline metals requires the generalized stacking fault energy (GSF) curve that was first introduced by Vitek ${ }^{20,21}$, involving both stable stacking fault energy $\gamma_{\text {sf }}$ and unstable stacking fault energy $\gamma_{\text {usf }}$.

Study on stacking fault energy can help to better understanding slip behavior in nanocrystalline materials and, thereby, to understand how to improve their mechanical properties. 
It is desirable to know the shape of the entire GSF curve to use it in a criterion for nucleation. Since only a single point know as the intrinsic (or stable) stacking fault $\gamma_{\mathrm{sf}}$ can be measured experimentally, many efforts to calculate this curve are based on modeling and simulation methods such as density functional theory (DFT) and molecular dynamics (MD). However, Most of the previous simulation works in calculating GSF are conducted in an undeformed or stress free crystal structures, which is far from the actual situation where the micro or nano-components can be deformed under multiple stress state. The preloading strain or stress on crystal structures are determined to significantly influence the GSF curve. For instance, Zimmerman et al. ${ }^{22}$ observed that the unrelaxed $\gamma_{\text {usf }}$ value of $175 \mathrm{~mJ} / \mathrm{m}^{2}$ was reduced to $99 \mathrm{~mJ} / \mathrm{m}^{2}$ after biaxially stretch the lattice by $4 \%$ when calculating the GSF curve of $\mathrm{Cu}$. Tschopp et al. ${ }^{23}$ used MD simulations to investigate the influence of normal stress on the GSF curve in $\mathrm{Cu}$, they found that the compressive (tensile) normal stress increases (decreases) the unstable stacking fault energy $\gamma_{\text {usf }}$, while the stable stacking fault energy $\gamma_{\mathrm{sf}}$ changes in an opposite manner. In addition, the stress influence on GSF curve is not only limited in the normal direction of the slip plane, lateral stress can also influence the value of generalized stacking fault energy. Ogata et al. ${ }^{24}$ used DFT calculation of stacking fault energy for $\mathrm{Al}$ and $\mathrm{Cu}$ to study their ideal shear strength. The results indicated that the hydrostatic pressure has a significant effect on the critical resolved shear stress at the atomic scale. Further, Tschopp et al. ${ }^{25,26}$ proposed that the stress required for dislocation nucleation depends on both Schmid stress component (resolved shear stress in the slip direction) and non-Schmid stress component (resolved normal stress and resolved shear stress perpendicular to the slip direction) acting on the $\left\{\begin{array}{lll}1 & 1 & 1\end{array}\right\}$ slip plane. Our previous work ${ }^{27,28}$ also showed that the effect of stress state can play an important role in dislocation nucleation and fracture of nanocrystalline $\mathrm{Cu}$.

All of the described works show that the GSF curve can be affected by the magnitude and directionality of the applied stress. In the previous study, Rice ${ }^{29}$ indicated that unstable stacking fault energy $\gamma_{\text {usf }}$ of the GSF curve was associated with the energy barrier for dislocation nucleation. Tadmor and $\mathrm{Hai}^{30,31}$ developed a criterion for the deformation mechanism of mechanical twinning, they found that the 'twinning tendency' was closely related to the unstable twin fault energy $\gamma_{\text {utf }}$ of the GSF curve. In this sense, the energy barrier of both dislocation nucleation and twinning formation in crystals can be influenced by the stress state of crystal lattice. The present work is carrying out MD simulations to investigate the effect of preloading stress with different direction and magnitude on the GSF curve of three fcc metals $(\mathrm{Cu}, \mathrm{Al}$ and $\mathrm{Ni})$. The unstable stacking fault energy $\left(\gamma_{\mathrm{usf}}\right)$, stable stacking fault energy $\left(\gamma_{\mathrm{sf}}\right)$ and unstable twin fault energy $\left(\gamma_{\text {utf }}\right)$ will be considered.

\section{Simulation method}

Simulations were performed by the parallel MD code LAMMPS ${ }^{32}$. The embedded atom method (EAM) potential was used in MD simulations. The EAM method defines the total energy of an elemental system which represented as:

$$
E_{t o t}=\frac{1}{2} \sum_{i \neq j} V\left(r_{i j}\right)+\sum_{i} F\left(\rho_{i}\right)
$$

Where $V\left(r_{i j}\right)$ is a pair potential as a function of distance $r_{i j}$ between atoms $i$ and $j . F\left(\rho_{i}\right)$ is the 
embedding energy as a function of the host electron density $\rho_{i}$ induced at site $i$ by all other atoms in the system. In this study, EAM potentials developed by Mishin et al. for $\mathrm{Cu}^{33}, \mathrm{Al}^{34}$, and $\mathrm{Ni}^{35}$ were used. These well-defined potentials were widely used in the MD simulations and they can fit a large set of experimental and first-principles data. For example, the intrinsic (stable) stacking fault energy $\gamma_{\text {sf }}$ and unstable stacking fault energy $\gamma_{\text {usf }}$ of $\mathrm{Cu}$ from their simulation result is 44.4 $\mathrm{mJ} / \mathrm{m}^{2}$ and $158 \mathrm{~mJ} / \mathrm{m}^{2}$ respectively ${ }^{33}$, which are very close to the experimental measurement 45 $\mathrm{mJ} / \mathrm{m}^{236}$ and $162 \mathrm{~mJ} / \mathrm{m}^{2}{ }^{33}$ Similarly for Al, Mishin et al. reported an intrinsic stacking fault energy of $146 \mathrm{~mJ} / \mathrm{m}^{2}$ and an unstable stacking fault energy of $168 \mathrm{~mJ} / \mathrm{m}^{2}$, both of which are in agreement with the $a b$ initio calculations performed in their work. ${ }^{34}$

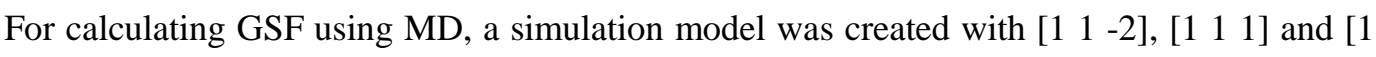
$-10]$ directions and it was divided into two blocks in the normal direction (see in Fig.1). A free boundary condition was used in the normal direction ([ $\left.\begin{array}{lll}1 & 1 & 1\end{array}\right]$ direction), while a periodic boundary

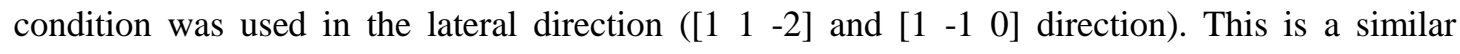
boundary condition used in Ref. ${ }^{37}$ to study the effect of vacancy defects on GSF energy of fcc metals. The GSF curve was determined by rigidly displacing the upper block on a $\left(\begin{array}{lll}1 & 1 & 1\end{array}\right)$ plane along a $\left[\begin{array}{lll}1 & 1 & -2\end{array}\right]$ direction while fixing the lower block and calculating the energy change in the whole simulation model. When displacing the upper block along [ [ $111-2]$ direction, the lateral motion of atoms was constrained. The LAMMPS code for calculating the GSF curve of $\mathrm{Cu}$ in this study under stress free condition is given in the Appendix section.

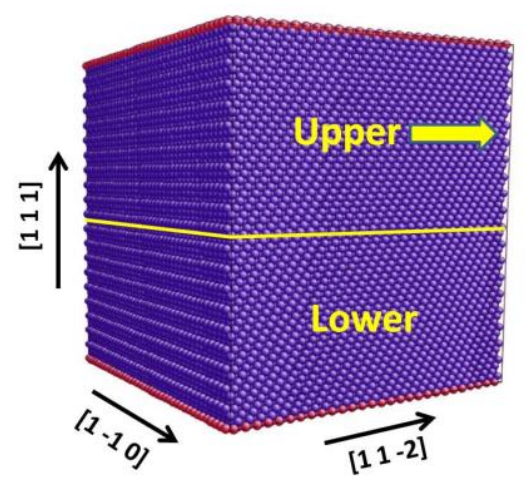

(a)

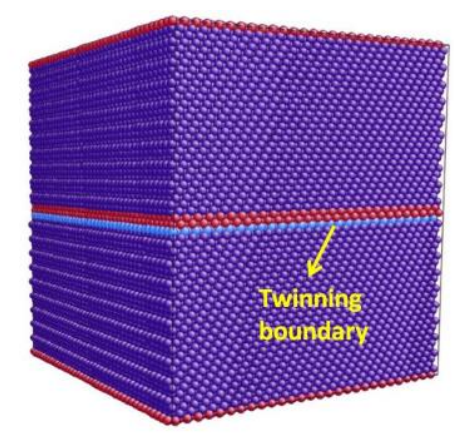

(b)

Figure.1 Simulation model for calculating the generalized stacking fault (GSF) energy curve. (a) The starting configuration with perfect fcc lattice. (b) A twinning boundary was formed after rigidly displacing the upper block on a $\left(\begin{array}{lll}1 & 1 & 1\end{array}\right)$ plane along a $\left[\begin{array}{lll}1 & 1 & -2\end{array}\right]$ direction. Atoms with perfect fcc structure are colored with dark blue, the red atoms represent the stacking fault and the free surface, the light blue atoms indicate the twin fault.

For the case of calculating $\gamma_{\mathrm{usf}}$ and $\gamma_{\mathrm{sf}}$, the starting configuration is a perfect fcc lattice ${ }^{22,}$ ${ }^{27}$ (see in Fig.2-a). Along the path, the system will have to first pass through an energy barrier that is referred as unstable stacking fault energy $\gamma_{\text {usf }}$, the position of the displaced atoms is shown in Fig.2(b). Zimmerman et al. ${ }^{22}$ indicated that the ideal displacement of the fcc lattice when $\gamma_{\text {usf }}$ reached equals to one-half of the partial Burgers vector $a_{0} / \sqrt{6}\left(a_{0}\right.$ is the equilibrium fcc lattice parameter). The simulation cell became stable when the displacement is $a_{0} / \sqrt{6}$, although the cell is not in its bulk equilibrium structure. The configuration in Fig.2(c) is known as the intrinsic

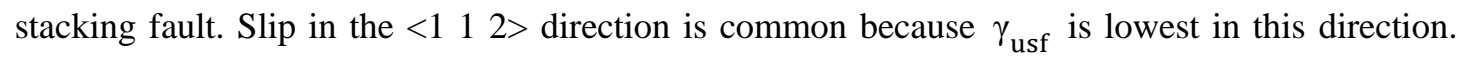
For the case of calculating $\gamma_{\text {utf }}$, the starting configuration is a pre-existing stacking fault. ${ }^{34}$ 
Specifically, $\gamma_{\text {utf }}$ was calculated by rigidly shifting the block along [ $\left[\begin{array}{lll}1 & 1 & -2\end{array}\right]$ direction in a $\left(\begin{array}{lll}1 & 1 & 1\end{array}\right)$ plane that is one atom layer above a stacking fault previously formed by shearing, as illustrated in Fig.2(d). Fig.1(b) shows the configuration of simulation cell when a twinning boundary was formed after rigidly displacing the upper block.

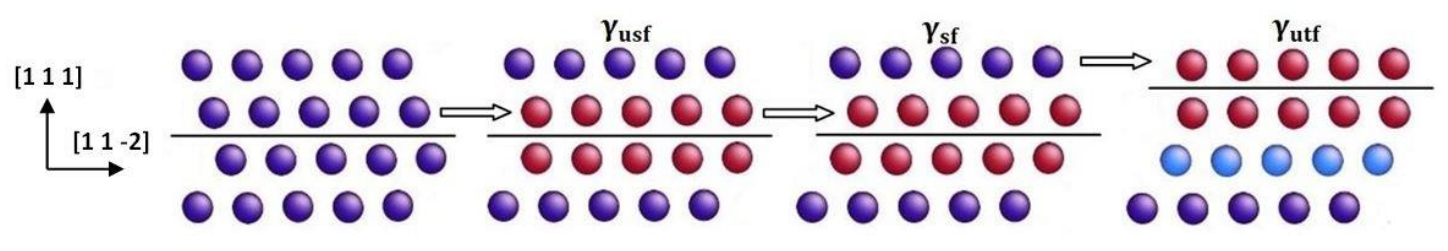

(a)

(b)

(c)

(d)

Figure.2 Schematic view of the atom positions when calculating the GSF curve. (a) perfect fcc crystal (b) unstable stacking fault (c) stable stacking fault, and (d) unstable twin fault. Configurations is viewed from the [ $\left.\begin{array}{lll}1 & -1 & 0\end{array}\right]$ direction. Atoms with perfect fcc structure are colored with dark blue, the red atoms represent the stacking fault and the light blue atoms represent the twin fault.

To simulate the influence of stress on the GSF curve, the simulation cell was uniformly strained along one of the following directions: [111], [11-2] and [1-10] with a constant rate of $10^{8} / \mathrm{s}$ at $1 \mathrm{~K}$ before the calculation of the GSF energy in the (111)[11-2] slip system. The high strain rate is inherent in the simulations for computational efficiency to have the desired amount of deformation within a given simulation time. An isobaric-isothermal (NPT) ensemble was used during the uniaxial tensile or compression. While the strain deformation was applied in one direction, the pressure of the lateral directions was kept zero. The system stress was attained by calculating the pressure of the entire system of atoms. The pressure was computed by the formula:

$$
P_{i j}=\frac{1}{V}\left[\sum_{k}^{N} m_{k} v_{k_{i}} v_{k_{j}}+\sum_{k}^{N} r_{k_{i}} f_{k_{j}}\right],(i, j=x, y, z)
$$

where the first term uses components of the kinetic energy tensor and the second term uses components of the virial tensor. $N$ is the total number of atoms in the simulation model, $V$ is the simulation model volume. $r$ and $f$ is the force vector and the distance vector respectively. System strain was derived from the positions of the periodic boundaries.

\section{Results and discussion}

Fig.3 plots the stress-strain response of the three investigated fcc metals $(\mathrm{Cu}, \mathrm{Al}$ and $\mathrm{Ni})$ under

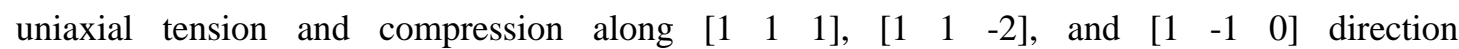
respectively. Notice that, the negative values of compressive stress are plotted in these graphs in order to compare with the values of tensile stress. Obviously, elastic modulus show big differences for different elements. Also, due to the material anisotropy, elastic modulus are slightly different for the same element in different directions. In addition, tension-compression asymmetry in elastic response is evident in all directions. In general, the elastic modulus of compression is higher than the value of tension, and this trend of asymmetry is more obvious for $\mathrm{Al}$ than the other two elements. The elastic modulus calculated from the initial slope of the MD simulations are listed in Table-1. Notice that, the nonlinear elastic effect is obvious for Al in [ [ $\left.\begin{array}{lll}1 & -1 & 0\end{array}\right]$ direction. This nonlinear stress-strain response is due to the non-negligible lattice rotation during elastic deformation at high strain ${ }^{23}$. 


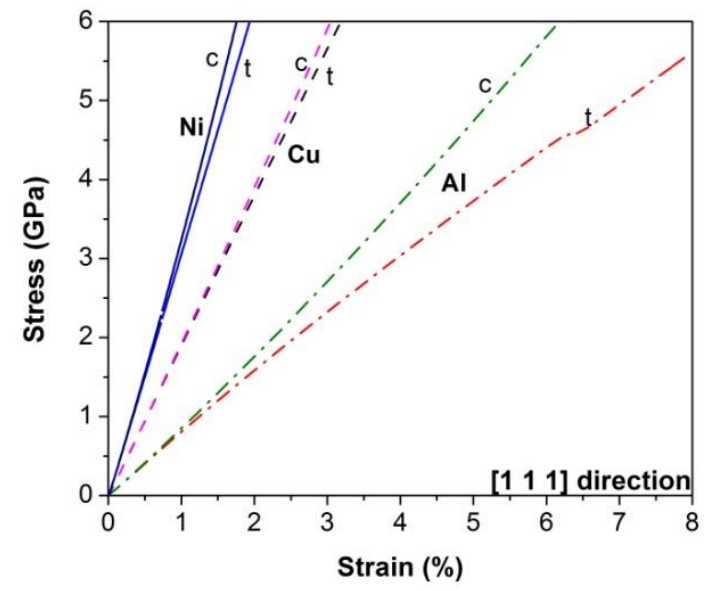

(a)

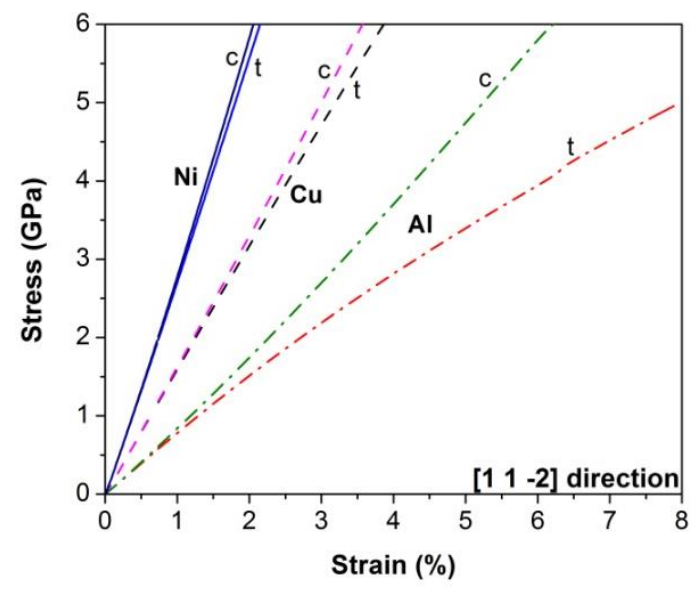

(b)

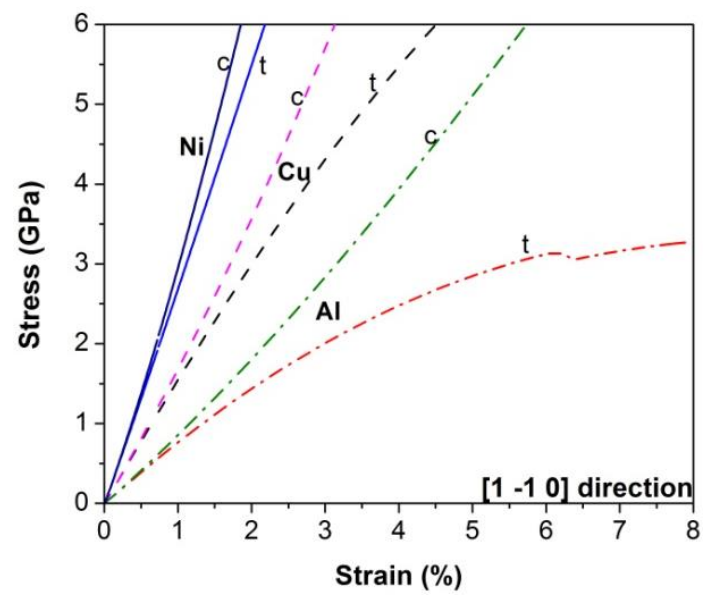

(c)

Figure.3 Stress-strain curves for uniaxial tension and compression of $\mathrm{Cu}, \mathrm{Al}$ and $\mathrm{Ni}$ along (a) [ $\left[\begin{array}{lll}1 & 1 & 1\end{array}\right]$ direction, (b)

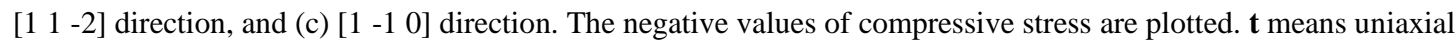
tension while $\mathbf{c}$ means uniaxial compression.

Table-1. Elastic modulus calculated from MD simulation

\begin{tabular}{ccccc}
\hline \multirow{2}{*}{ Element } & Preloading & $\mathbf{E}_{[\mathbf{1 1 1}]}$ & $\mathbf{E}_{[\mathbf{1 1 ~ 2}]}$ & $\mathbf{E}_{[\mathbf{1 ~ 1 ~ 1 ~ 0 ] ~}}$ \\
\hline \multirow{2}{*}{$\mathrm{Cu}$} & Tension & 189.8 & 159.1 & 154.4 \\
& Compression & 192.3 & 162.0 & 167.9 \\
\multirow{2}{*}{$\mathrm{Ni}$} & Tension & 305.4 & 270.4 & 268.2 \\
& Compression & 323.4 & 277.3 & 294.8 \\
$\mathrm{Al}$ & Tension & 80.7 & 78.0 & 76.1 \\
& Compression & 84.9 & 83.7 & 85.3 \\
\hline
\end{tabular}

The influence of the preloading stress on the GSF curve of $\mathrm{Cu}$ is shown in Fig.4. In the normal [ $\left[\begin{array}{lll}1 & 1 & 1\end{array}\right]$ direction and lateral [ $\left[\begin{array}{lll}1 & -1 & 0\end{array}\right]$ direction, the tensile (compressive) stress decreases (increases) the unstable stacking fault energy $\left(\gamma_{\mathrm{usf}}\right)$ and the unstable twin fault energy $\left(\gamma_{\mathrm{utf}}\right)$, the greater the magnitude of the preloading stress, the greater the decreases (increases) of the value. However, the stable stacking fault energy $\left(\gamma_{\mathrm{sf}}\right)$ changes in an opposite manner in $\left[\begin{array}{lll}1 & 1 & 1\end{array}\right]$ direction. $\gamma_{\mathrm{sf}}$ increases under the preloading tensile stress and decreases under the compressive stress. The effect is more noticeable at higher stress of compression. For example, $\gamma_{\mathrm{sf}}$ increases $4.9 \%$ at 5 GPa tensile stress and decreases $17.5 \%$ at $5 \mathrm{GPa}$ compressive stress. Different from the cases in [1 
11 and [ [ $\left.\begin{array}{lll}1 & -1 & 0\end{array}\right]$ directions, the tensile stress increase the value of $\gamma_{u s f}$ and $\gamma_{u t f}$ in $\left[\begin{array}{lll}1 & 1 & -2\end{array}\right]$ direction while the compressive stress decreases the values. It is interesting to note that, under the preloading stress in all directions, the value of $\gamma_{\mathrm{usf}}, \gamma_{\mathrm{sf}}$ and $\gamma_{\mathrm{utf}}$ may reached before or after their ideal displacement, a value that can be expected from geometric considerations. Ideally in the figures, $\delta_{\mathrm{sf}}=\frac{1}{2} \mathrm{a}_{0} / \sqrt{6}, \delta_{\text {usf }}=\mathrm{a}_{0} / \sqrt{6}, \delta_{\text {utf }}=\frac{3}{2} \mathrm{a}_{0} / \sqrt{6}$, where $\mathrm{a}_{0}$ is the equilibrium fcc lattice parameter. This deviation is mainly due to the pre-strain changes the interatomic distance of the equilibrium structure.

Fig.5 shows the effect of stress on the GSF curve of Ni. In general, the influence of tensile and compressive stress on the GSF curve of $\mathrm{Ni}$ in all directions is similar to the stress effect on $\mathrm{Cu}$. In $\left[\begin{array}{lll}1 & 1 & 1\end{array}\right]$ and $\left[\begin{array}{lll}1 & -1 & 0\end{array}\right]$ directions, $\gamma_{\text {usf }}$ and $\gamma_{\text {utf }}$ decreases (increases) with the increased magnitude of tensile and compressive stress, while the tensile and compressive stress effect in an opposite way in [ $\left.\begin{array}{lll}1 & 1 & -2\end{array}\right]$ direction. The influence of the preloading stress on GSF curve of $\mathrm{Ni}$ is not as obvious as that of $\mathrm{Cu}$, and the deviation of the ideal displacement when each value reached is less than that of $\mathrm{Cu}$. This is due to the higher elastic modulus of $\mathrm{Ni}$ than $\mathrm{Cu}$ (see in Fig.3), i.e. lattice deformation and the change of the interatomic distance is less in $\mathrm{Ni}$ than $\mathrm{Cu}$ at the same value of the applied stress.

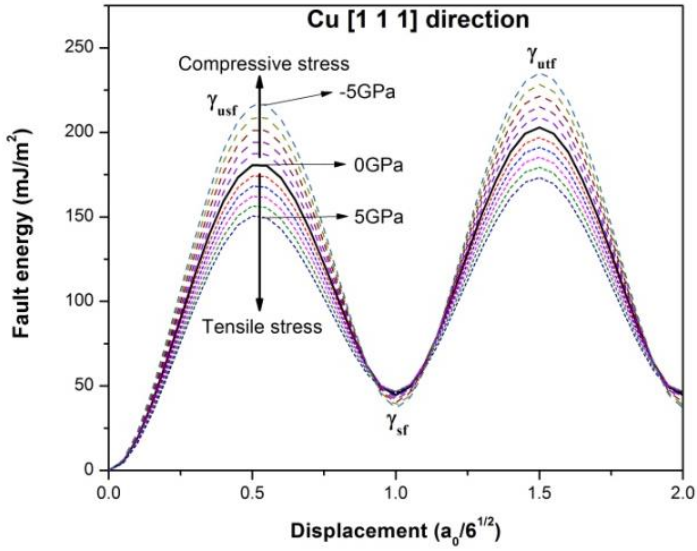

(a)

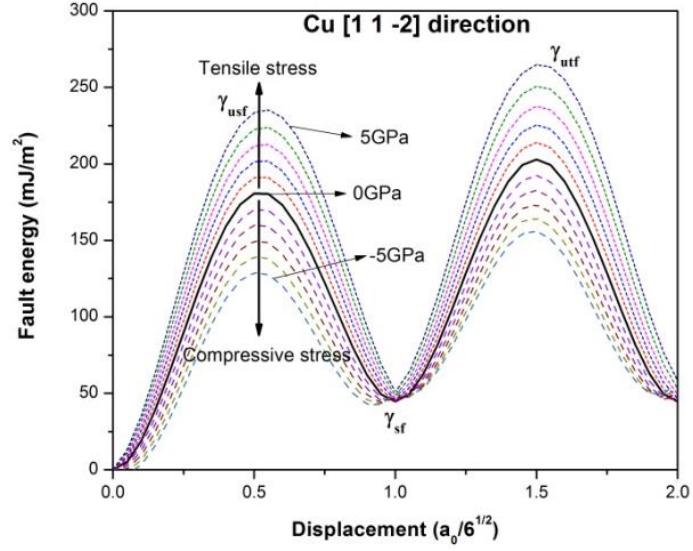

(b)

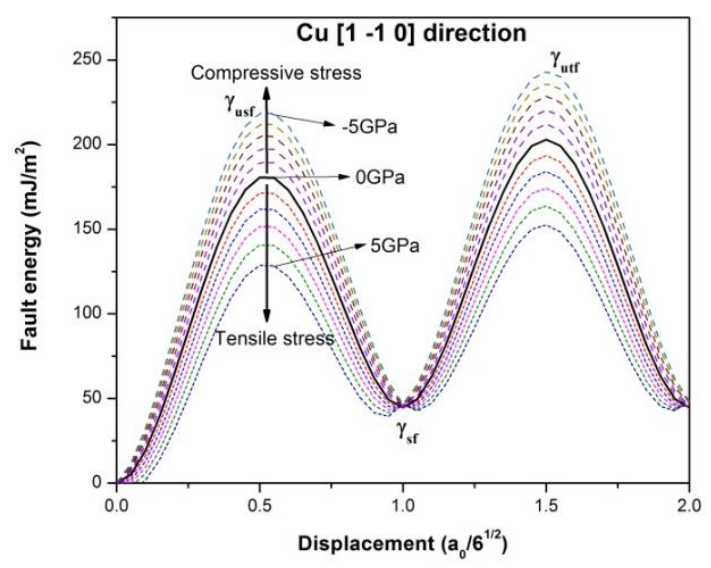

(c)

Figure.4 Influence of stress on the generalized stacking fault energy curve in $\mathrm{Cu}$. The tensile and compressive

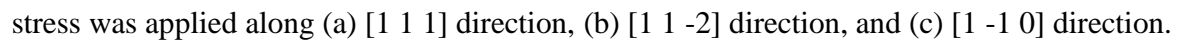




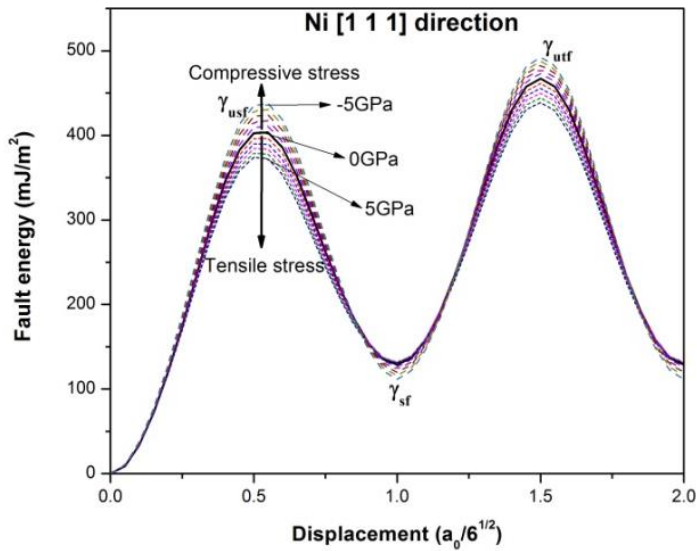

(a)

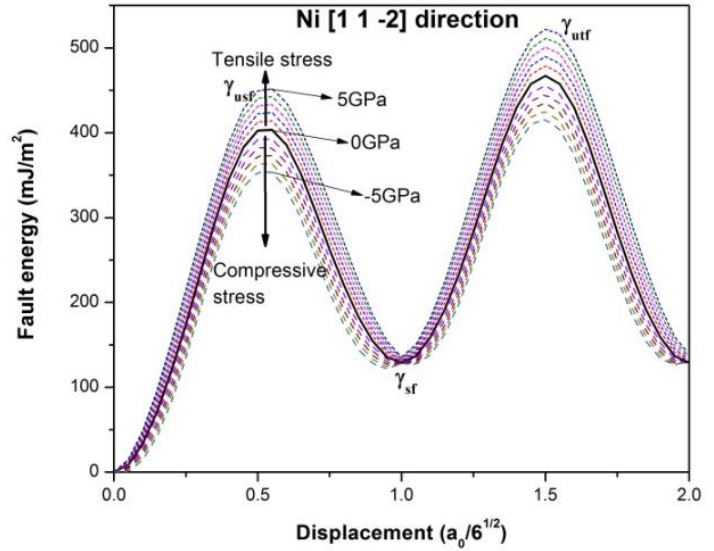

(b)

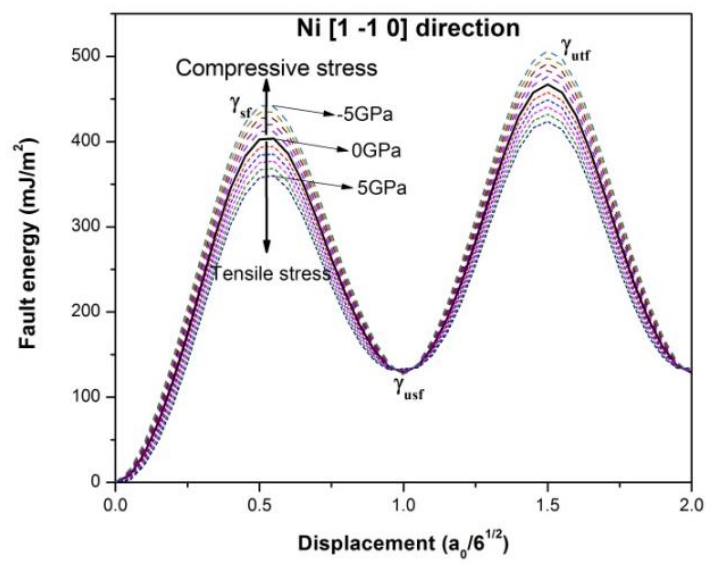

(c)

Figure.5 Influence of stress on the generalized stacking fault energy curve in $\mathrm{Ni}$. The tensile and compressive

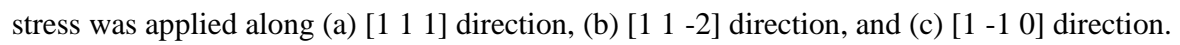

For $\mathrm{Cu}$ and $\mathrm{Ni}$, the preloading stress has an obvious influence on the value of $\gamma_{\text {usf }}$ and $\gamma_{\text {utf }}$, while the influence on the value of $\gamma_{\mathrm{sf}}$ is limit. However, this is not the case of $\mathrm{Al}$, the preloading stress can greatly influence on the three values in all directions, especially in the normal [ $\left[\begin{array}{lll}1 & 1 & 1\end{array}\right]$ direction. Fig. 6 shows the influence of tensile and compressive stress on the GSF curve of Al. In Fig.6(a), the value of $\gamma_{\mathrm{usf}}, \gamma_{\mathrm{sf}}$ and $\gamma_{\mathrm{utf}}$ increases for $41.2 \%, 59.8 \%$ and $43.3 \%$ at $5 \mathrm{GPa}$ compressive stress and drops sharply for $53.5 \%, 82.7 \%$ and $59.4 \%$ respectively at $5 \mathrm{GPa}$ tensile stress. Another difference of $\mathrm{Al}$ from $\mathrm{Cu}$ and $\mathrm{Ni}$ in the GSF curve is observed in [ $1-10]$ direction. In Fig.4(c) and Fig.5(c), $\gamma_{\text {usf }}$ and $\gamma_{\text {utf }}$ decrease in tension and increase in compression, while in Fig.6(c), this effect acts in an opposite manner. In addition, the applied tension stress plays a little

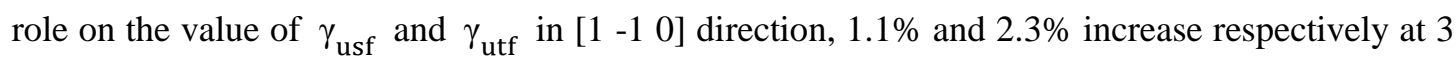
$\mathrm{GPa}$ tensile stress. Moreover, the value of different fault energies can changes faster at a higher tensile stress in [ $\left[\begin{array}{lll}1 & 1 & -2\end{array}\right]$ and [ $\left[\begin{array}{lll}1 & -1 & 0\end{array}\right]$ directions due to the nonlinear elastic effect in these directions of $\mathrm{Al}$. 


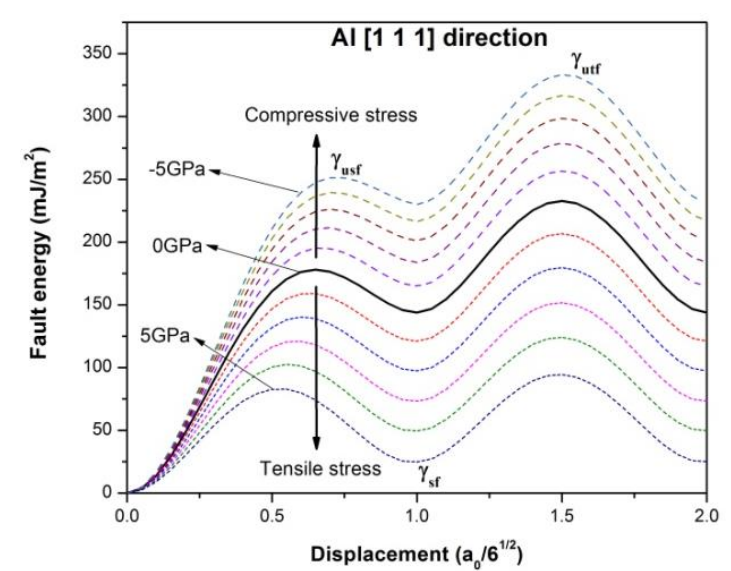

(a)

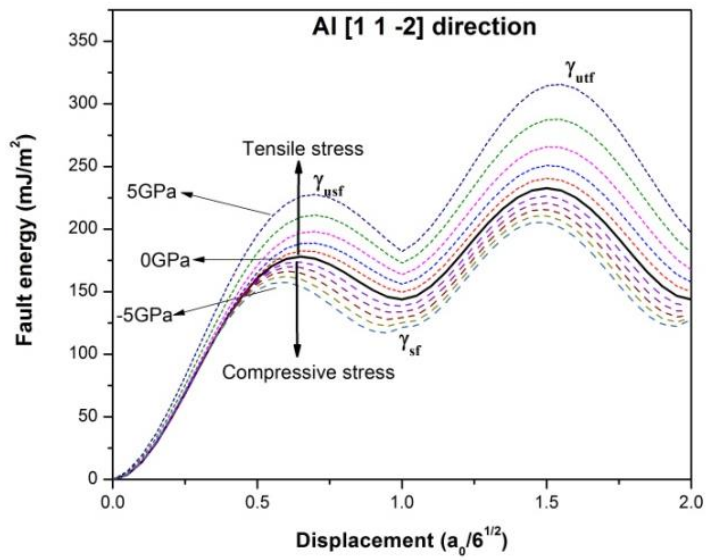

(b)

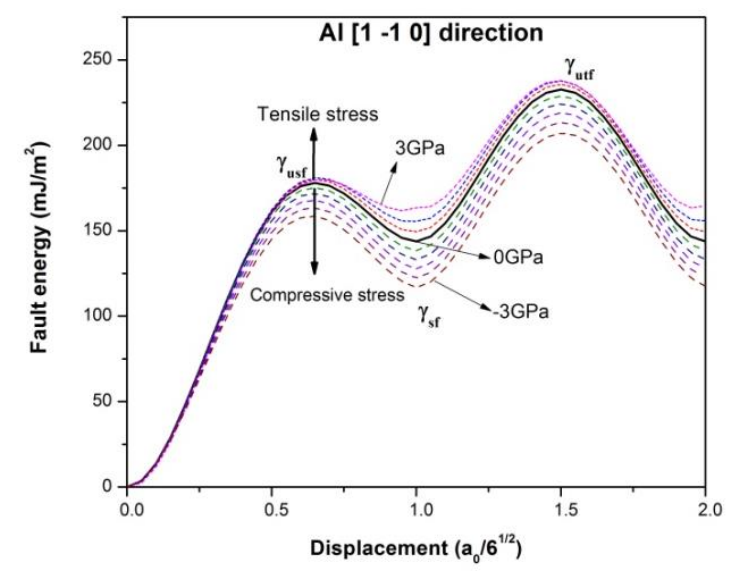

(c)

Figure.6 Influence of stress on the generalized stacking fault energy curve in Al. The tensile and compressive

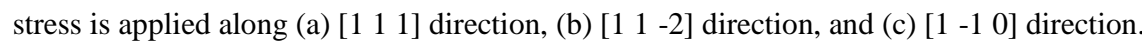

In previous studies, MD simulations have revealed that the small gain size in nanocrystalline materials can result in the heterogeneous nucleation and emission of dislocations from the GBs. The deformation mechanism has been confirmed by recent in situ transmission electron microscopy experiments in nanocrystalline $\mathrm{Al}^{38}$ and $\mathrm{Cu}^{39}$. The experiments have also shown that stacking faults and deformation twins can be formed through partial dislocation emission from GBs. Van Swygenhoven et al. ${ }^{19}$ indicated that all aspects of the GSF curve have to be incorporated to well understand the slip activities observed in simulations, the deformation cannot be explained by means of the absolute value of $\gamma_{\text {sf }}$ alone as suggested by Yamakov et al. ${ }^{18}$. Specifically, whether extended partial dislocations or full dislocations travelling through the grains dominates the deformation mechanism in a simulation must understood in terms of the ratio $\gamma_{\text {sf }} / \gamma_{\text {usf }}$. This value closer to unity is associated with fec materials that nucleate full dislocations.

Fig.7 shows the ratio $\gamma_{\text {sf }} / \gamma_{\text {usf }}$ changes as a function of the preloading stress in different directions. For the case of $\mathrm{Cu}$ and $\mathrm{Ni}$, the applied tensile stress increases the value while the

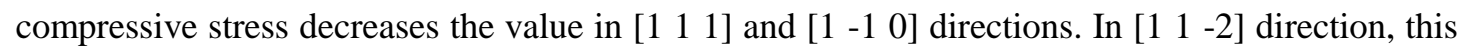
effect acts in an opposite manner. Overall, the influence of the applied stress on the value of $\gamma_{\text {sf }} / \gamma_{\text {usf }}$ for $\mathrm{Cu}$ and $\mathrm{Ni}$ is not obvious. The value is at a relatively low level (ranged from 0.17 to 0.33 for $\mathrm{Cu}$ and 0.26 to 0.37 for $\mathrm{Ni}$ ), which means the nucleation of partial dislocation and its propagation in the grain is still the dominant mechanism in nanocrystalline $\mathrm{Cu}$ and $\mathrm{Ni}$ even in a 
condition of high stress concentration.

For the case of $\mathrm{Al}$, the ratio $\gamma_{\mathrm{sf}} / \gamma_{\mathrm{usf}}$ decreases significantly with the increase of the preloading tensile stress in [ [ $\left.\begin{array}{lll}1 & 1 & 1\end{array}\right]$ direction (see in Fig.7-a). At 5 GPa tensile stress, the ratio drops to the same level as $\mathrm{Cu}$ and $\mathrm{Ni}$. The sharp decrease of $\gamma_{\mathrm{sf}} / \gamma_{\mathrm{usf}}$ indicates that the nucleation of full dislocation (with trailing partial dislocation) that observed in simulations of $\mathrm{Al}^{17,19}$ may be restricted under tensile stress that applied normal to the slip plane, i.e. the extended partial dislocation may dominate the deformation mechanisms as same as the case of $\mathrm{Cu}$ and $\mathrm{Ni}$. In

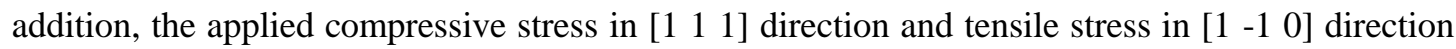
increases the ratio $\gamma_{\mathrm{sf}} / \gamma_{\text {usf }}$ to a level more closer to unity, as shown in Fig.7(a) and (c). It means that the trailing partial dislocations can nucleate more easily and the separation of the leading and trailing partials will be shortened. The effect of stress in [lll $11-2]$ direction on the ratio $\gamma_{\mathrm{sf}} / \gamma_{\mathrm{usf}}$ is not obvious, ranged from 0.74 to 0.83 .

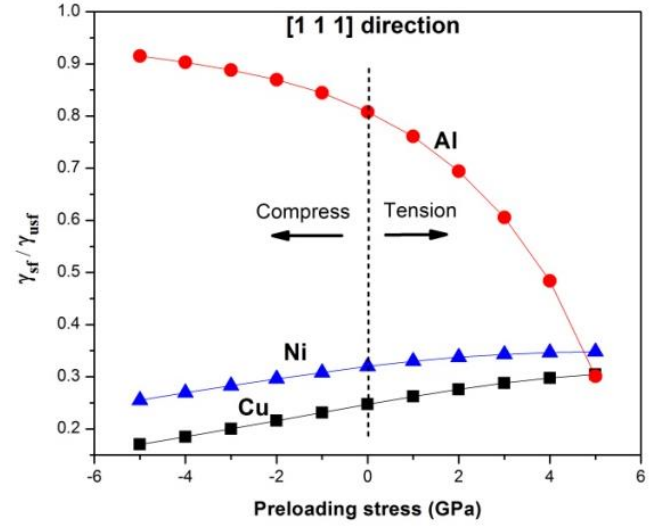

(a)

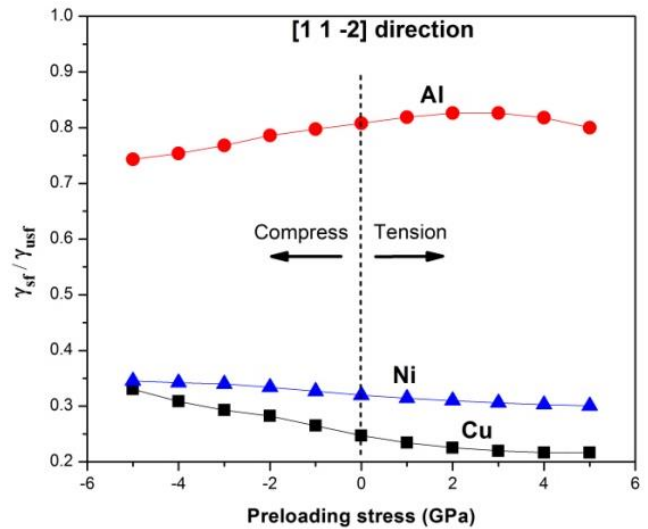

(b)

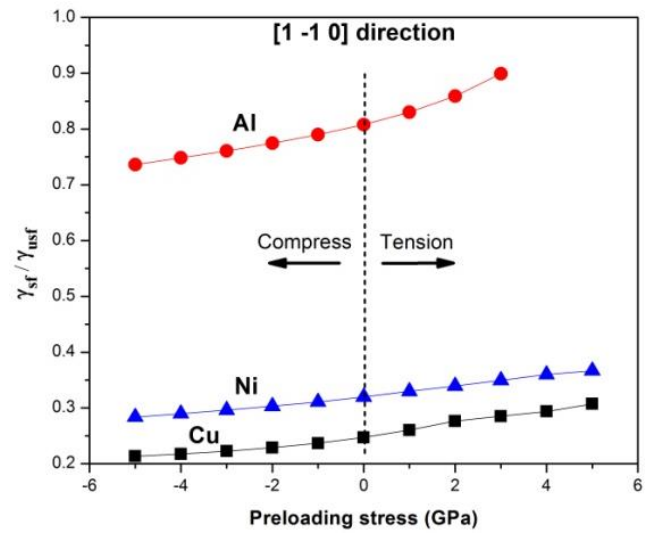

(c)

Figure.7 The ratio of stable stacking fault energy to unstable stacking fault energy $\left(\gamma_{\text {sf }} / \gamma_{\text {usf }}\right)$ as a function of the

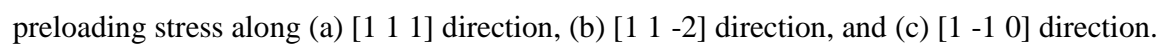

As previously reported ${ }^{30,31}$, the observation of twinning nucleation in a simulation is depend on the ratio $\gamma_{\text {utf }} / \gamma_{\text {usf }}$, where $\gamma_{\text {utf }}$ relates to the energy barrier for twinning formation and $\gamma_{\text {usf }}$ is associated with the barrier for a full dislocation nucleation. The ratio controls the competition of the two possible mechanisms. $\gamma_{\text {utf }}$ is larger than $\gamma_{\text {usf }}$ in all cases of our simulation for the GSF curve, which is consistent with the results of Van Swygenhoven et al. ${ }^{19}$ by testing the GSF curves with different EAM potentials. This can explain why mechanical twinning is not observed as the dominant mechanisms in most of the MD simulations of nanocrystalline samples with defect-free 
grains, since it will overcome greater energy barrier. The influence of the applied stress on the ratio $\gamma_{\text {utf }} / \gamma_{\text {usf }}$ is shown in Fig.8. In general, the value of $\gamma_{\text {utf }} / \gamma_{\text {usf }}$ for $\mathrm{Cu}$ is at the lowest level of all the three tested elements, and this value is more closer to unity when compressive stress applied normal to the slip plane (see in Fig.8-a). It means that the barrier for twinning formation and for dislocation nucleation is comparable and twinning is easier to grow in $\mathrm{Cu}$ in certain conditions. For example, $\mathrm{Lu}$ and collaborates ${ }^{40}$ used the nanoscale growth twins in $\mathrm{Cu}$ sample to

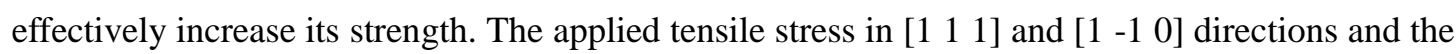
compressive stress in [1 1-2] direction increases the value of $\gamma_{\text {utf }} / \gamma_{\text {usf }}$ for $\mathrm{Cu}$ so that increases the difficulty of twinning formation. For the case of $\mathrm{Ni}$, the effect of stress on the ratio $\gamma_{\mathrm{utf}} / \gamma_{\mathrm{usf}}$ is not significant in all directions, this value ranges from 1.13 to 1.18 .

The value of $\gamma_{\mathrm{utf}} / \gamma_{\mathrm{usf}}$ for $\mathrm{Al}$ is much higher than the value of $\mathrm{Cu}$ and $\mathrm{Ni}$, so the twinning is more difficult to form in Al. However, the tensile stress in [ [ 1111$]$ direction can sharply decrease the value, from 1.31 in stress free condition to 1.14 at 5 GPa preloading stress. The sharp decrease of $\gamma_{\text {utf }} / \gamma_{\text {usf }}$ indicates that twinning may also observed as a deformation mechanism in high stress concentration condition, especially when the applied tensile stress has a high component normal to the slip plane. For example, twinning deformation mechanism has been observed in the experiments $^{38,39}$, in which twin boundary nucleation becomes favorable over full dislocation nucleation when high shear stress are provided (for example, during ball milling and high-pressure

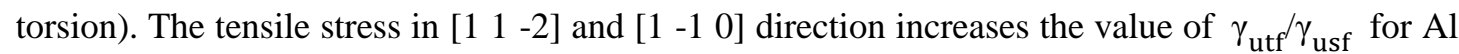
so that can increase the difficulty of twinning formation.

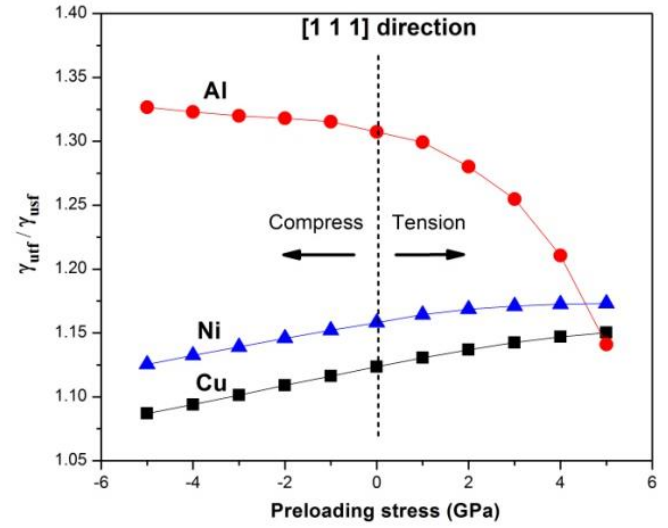

(a)

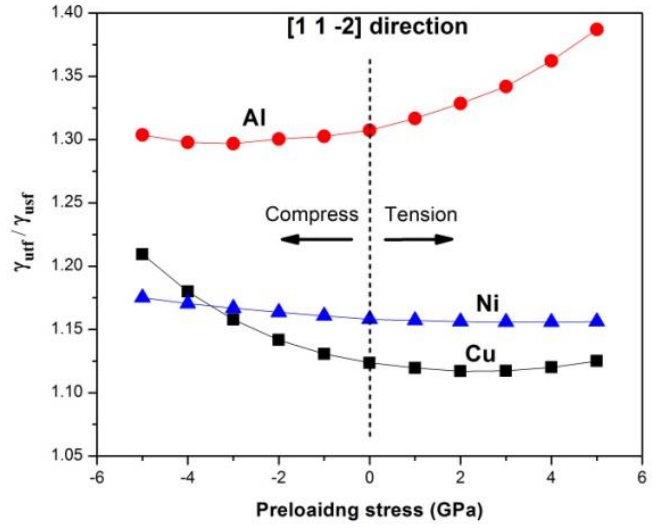

(b)

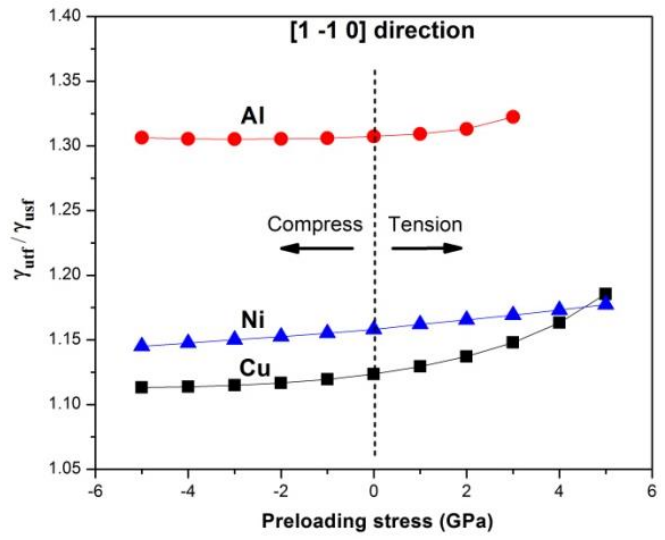

(c)

Figure.8 The ratio of unstable twin fault energy to unstable stacking fault energy $\gamma_{\text {utf }} / \gamma_{\text {usf }}$ as a function of the

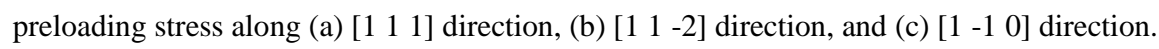




\section{Conclusion}

MD simulations were used to investigate the influence of tensile and compressive stress on (1

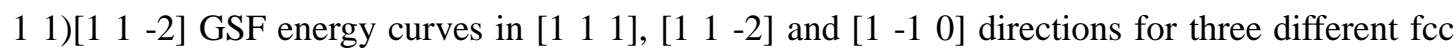
metals. The widely used EAM potentials developed by Mishin for $\mathrm{Cu}^{33}, \mathrm{Al}^{34}$, and $\mathrm{Ni}^{35}$ were tested in this study. Simulation results show that GSF curves of the three elements can be influenced by the preloading stress. The value of $\gamma_{\mathrm{usf}}, \gamma_{\mathrm{sf}}$ and $\gamma_{\mathrm{utf}}$ increased or decreased when preloading tensile or compressive stress was applied in certain direction. The effect of stress on the ratio of $\gamma_{\mathrm{sf}} / \gamma_{\mathrm{usf}}$ and $\gamma_{\mathrm{utf}} / \gamma_{\mathrm{usf}}$, which are closely related to the deformation mechanisms in nanocrystalline materials, were also investigated in this study. The results quantitatively demonstrate that not only the resolved stress along the slip plane (traditional Schmid factor), but also the resolved stress normal to the slip plane and the resolved stress perpendicular to the slip direction can play an important role in dislocation nucleation and twinning formation. The change in the GSF energy curve as a function of the applied stress indicates that the stress state is another important factor that can influence the deformation mechanisms of nanocrystalline materials.

\section{Acknowledgements}

L. Zhang, X. Zhao and L.Q. Pei would like to acknowledge the financial support from China Scholarship Council (CSC).

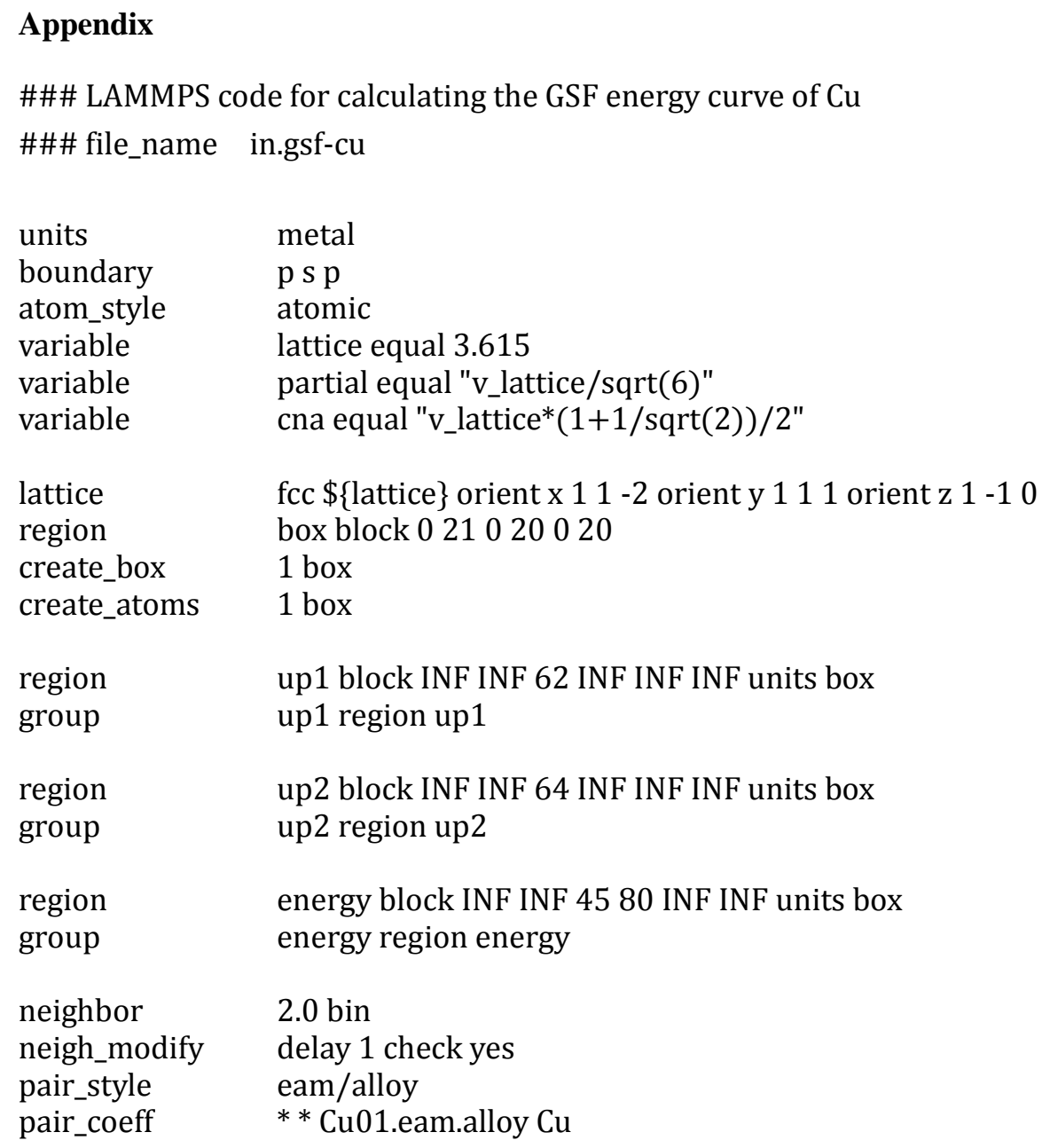




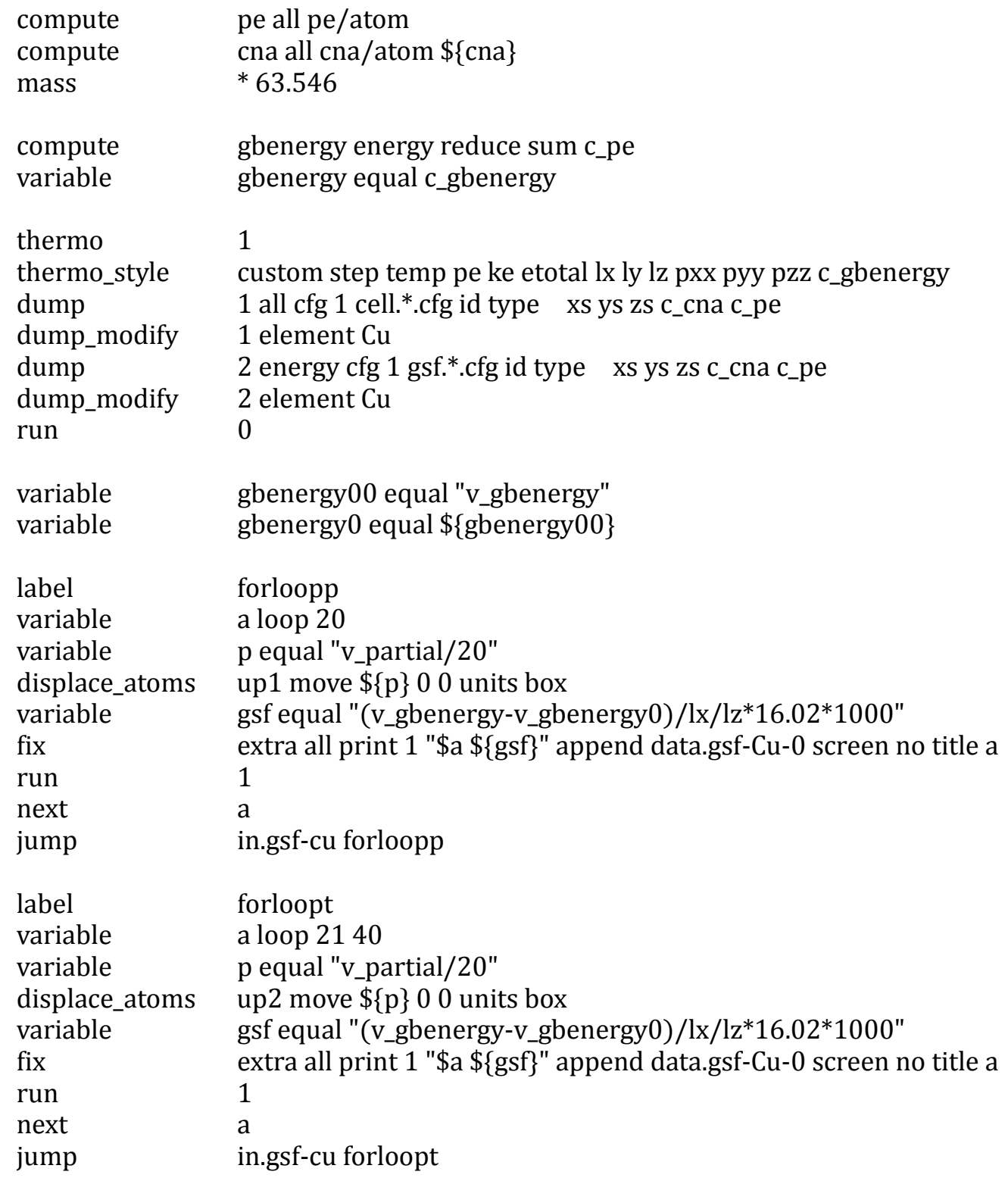

\section{References}

1. Champion Y, Langlois C, Guérin M S, Langlois P, Bonnentien J L and Hÿtch M J 2003 Science 300 310

2. Lu L, Shen Y, Chen X, Qian L and Lu K 2004 Science 304422

3. Ma E 2003 Nature Mater. 27

4. Schiøtz J and Jacobsen K W 2003 Science 3011357

5. Zhou Y, Erb U, Aust K T and Palumbo G 2003 Scr. Mater. 48825

6. Wang Y M, Hamza A V and Ma E 2005 App. Phys. Lett. 86241917

7. Wang Y M, Hamza A V and Ma E 2006 Acta Mater. 542715

8. Schiøtz J, Tolla F D and Jacobsen K W 1998 Nature 391561

9. Schiøtz J, Vegge T, Tolla F D and Jacobsen K W 1999 Phys. Rev. B 6011971

10. Schiøtz J 2004 Scr. Mater. 51837

11. Asaro R J, Krysl P and Kad B 2003 Philos. Mag. Lett. 83733

12. Koch C C 2003 Scr. Mater. 49657 
13. Kumar K S, Van Swygenhoven H and Suresh S 2003 Acta Mater. 515743

14. Van Swygenhoven H 2002 Science 29666

15. Tschopp M A and McDowell D L 2008 Int. J. Plast. 24191

16. Spearot D E, Jacob K I and McDowell D L 2007 Int. J. Plast. 23143

17. Spearot D E, Jacob K I and McDowell D L 2005 Acta Mater. 533579

18. Yamakov V, Wolf D, Phillpot S R, Mukherjee A K and Gleiter H 2004 Nature Mater. 343

19. Van Swygenhoven H, Derlet P M and Frøseth A G 2004 Nature Mater. 3399

20. Vítek V 1966 Phys. Status Solidi 18687

21. Vítek V 1968 Philos. Mag. 18773

22.Zimmerman J A, Gao H and Abraham F F 2000 Modell. Simul. Mater. Sci. Eng. 8103

23. Tschopp M A and McDowell D L 2008 J. Mech. Phys. Solids. 561806

24. Ogata S, Li J and Yip S 2002 Science 298807

25. Tschopp M A, Tucker G J and McDowell D L 2008 Comput. Mater. Sci. 44351

26. Spearot D E, Tschopp M A, Jacob K I and McDowell D L 2007 Acta Mater. 55705

27. Zhang L, Lu C and Tieu K 2014 Sci. Rep. 45919

28. Zhang L, Lu C, Tieu K, Pei L Q and Zhao X 2014 Chin. Phys. B 2398102

29. Rice J R 1992 J. Mech. Phys. Solids. 40239

30. Hai S and Tadmor E B 2003 Acta Mater. 51117

31. Tadmor E B and Hai S 2003 J. Mech. Phys. Solids. 51765

32. Plimpton S 1995 J. Comput. Phys. 1171

33. Mishin Y, Mehl M J, Papaconstantopoulos D A, Voter A F and Kress J D 2001 Phys. Rev. B 63 2241061

34. Zope R R and Mishin Y 2003 Phys. Rev. B 68024102

35. Mishin Y, Farkas D, Mehl M J and Papaconstantopoulos D A 1999 Phys. Rev. B 593393

36. Carter C B and Ray I L F 1977 Philos. Mag. 35189

37. Ebrahim A., Mohsen Asle Z, Amitava M and Mark A T 2014 J. Phys.:Condensed Matter. 26115404

38. Liao X Z, Zhou F, Lavernia E J, He D W and Zhu Y T 2023 Appl. Phys. Lett. 755062

39.Liao X Z, Zhao Y H, Srinivasan S G, Zhu Y T, Valiev R Z and Gunderov D V 2004 Appl. Phys. Lett. 75592

40. Lu K, Lu L, Chen X and Huang X 2009 Science 323607 